\title{
Inferring ancestry from population genomic data and its applications
}

\author{
Badri Padhukasahasram* \\ Center for Health Policy and Health Services Research, Henry Ford Health System, Detroit, MI, USA
}

\section{Edited by:}

Jeffrey Jensen, École Polytechnique

Fédérale de Lausanne, Switzerland

Reviewed by:

Joanna Kelley, Stanford University,

USA

Susanne Pfeifer, Max F. Perutz

Laboratories, Austria

\section{${ }^{*}$ Correspondence:}

Badri Padhukasahasram, Center for Health Policy and Health Services

Research, Henry Ford Health System,

1 Ford Place 3A, Detroit,

MI 48202, USA

e-mail: bpadhuk1@hfhs.org
Ancestry inference is a frequently encountered problem and has many applications such as forensic analyses, genetic association studies, and personal genomics. The main goal of ancestry inference is to identify an individual's population of origin based on our knowledge of natural populations. Because both self-reported ancestry in humans or the sampling location of an organism can be inaccurate for this purpose, the use of genetic markers can facilitate accurate and reliable inference of an individual's ancestral origins. At a higher level, there are two different paradigms in ancestry inference: global ancestry inference which tries to compute the genome-wide average of the population contributions and local ancestry inference which tries to identify the regional ancestry of a genomic segment. In this mini review, I describe the numerous approaches that are currently available for both kinds of ancestry inference from population genomic datasets. I first describe the general ideas underlying such inference methods and their relationship to one another. Then, I describe practical applications in which inference of ancestry has proven useful. Lastly, I discuss challenges and directions for future research work in this area.

Keywords: global ancestry, local ancestry, hidden Markov models, Bayesian inference, maximum likelihood estimation

\section{INTRODUCTION}

In population genomic analyses, it is often necessary to classify a sample of organisms into different population groups. This can inform us about the evolutionary relationships and migration history of natural populations and help identify an individual's population of origin. Because both the sampling location of an organism or self-reported ancestry in the case of humans can be uninformative for this purpose, the use of genetic markers can facilitate accurate and reliable ancestry inference by exploiting allele frequency differences across population groups. Recent advances in genomic technologies as well as computing resources have made it possible to accurately infer overall ancestry as well as ancestry at a fine-scale across an individual's genome. Ancestry estimation is a frequently encountered problem and has been used in a variety of applications such as tracing someone's geographic origin in forensic investigations, correcting for population stratification in genome-wide association studies and developing personalized approaches to treatment.

There are currently two different paradigms underlying ancestry inference: global ancestry (GA) estimation and local ancestry (LA) estimation. GA inference involves estimating the proportion of ancestry contributed by different populations averaged across the entire genome. Such methods have been applied to study population structure in humans (e.g., Pritchard et al., 2000; Rosenberg etal., 2002; Tang et al., 2005; Price et al., 2006; Lao et al., 2014) as well as in many other species (e.g., Nordborg et al., 2005; Becquet et al., 2007). In contrast, in LA inference, we interpret each chromosome in an individual's genome as a mosaic of segments that originate from different ancestral populations and the goal is to find the ancestral population of origin at each position. LA inference methods (e.g., Tang et al., 2006; Sankararaman et al., 2008; Maples et al., 2013) have been used mainly to study recently admixed populations such as African Americans and Latinos.

In this mini review, I will describe the various methods that are currently available for efficient and accurate inference of GA and LA from large genomic datasets. I first discuss the general ideas behind the different approaches that are used, their relationship to one another, relative performance in terms of speed and accuracy, advantages, and drawbacks. Then, I will describe many applications in which ancestry inference methods have proven to be useful. Lastly, I will discuss challenges and possible directions for future research in this area.

\section{METHODS FOR GLOBAL ANCESTRY ESTIMATION}

The main goal of GA inference is to estimate the fraction of ancestry contributed by each population as averaged across the entire genome. There are two broad categories of methods available for such inference: model-based approaches and non-parametric approaches.

\section{MODEL-BASED APPROACHES}

Model-based approaches for GA inference attempt to estimate individual ancestry coefficients assuming particular statistical models. For example, the programs STRUCTURE (Pritchard et al., 2000) and ADMIXTURE (Alexander etal., 2009) both model the probability of observed genotypes using ancestry proportions and population allele frequencies assuming Hardy-Weinberg equilibrium and linkage equilibrium among loci. STRUCTURE is based on a Bayesian approach that uses a Markov Chain 
Monte Carlo algorithm to obtain samples from the posterior distribution. Falush etal. (2003) later extended this method to allow for admixture linkage disequilibrium (LD). InStruct is an extension of STRUCTURE which can jointly infer both population structure and inbreeding rates for organisms that undergo self-fertilization (e.g., plants). The method relaxes the HardyWeinberg equilibrium assumption within clusters (Gao et al., 2007). fastSTRUCTURE uses efficient algorithms to infer posteriors underlying the STRUCTURE model employing a variational Bayesian framework. In this framework, posterior inference is posed as an optimization problem (Raj et al., 2014). ADMIXTURE employs the same model (Alexander et al., 2009) as STRUCTURE but uses a maximum likelihood estimation procedure involving high-dimensional optimization algorithms. In particular, this is accomplished through a block relaxation scheme that alternates between updating the ancestry coefficient matrix and population allele frequency matrix. Convergence is accelerated via a novel quasi-Newton method. ADMIXTURE is over an order of magnitude faster than STRUCTURE and produces estimates of similar accuracy (Alexander et al., 2009). The program FRAPPE (Tang et al., 2005) also follows the same likelihood model as STRUCTURE but estimates parameters by maximum likelihood estimation using an Expectation Maximization algorithm. Enforcing strict convergence criteria makes this program computationally burdensome. Therefore, in practice, relaxed convergence criteria are used which makes the results slightly less accurate than ADMIXTURE (Alexander et al., 2009). More recently, Frichot et al., 2014 described fast computational algorithms for ancestry inference that make use of sparse non-negative matrix factorization (sNMF) and least squares optimization to produce estimates of ancestry proportions. In particular, these algorithms produce ancestry estimates that are as accurate as ADMIXTURE but are $\sim 10-30$ times faster in terms of speed. SPA (spatial ancestry analysis) is a recently developed alternate approach that uses explicit probabilistic models for the change in allele frequency in space and uses these to position individuals into two or three dimensional space (Yang etal., 2012).

\section{NON-PARAMETRIC APPROACHES}

Non-parametric methods make use of techniques from multivariate analysis such as cluster analysis and principal component analysis (PCA) to infer structure in the data. The main goal of cluster analysis is to directly find subsets representing the different population groups in the data (e.g., Gao and Starmer, 2007; Lee et al., 2009; Bouaziz et al., 2012). Other techniques such as PCA (Patterson et al., 2006; Price et al., 2006), multidimensional scaling (MDS; Purcell et al., 2007), and principal coordinate analysis seek to construct projections in lower dimensional space that capture a large fraction of the variation in the marker genotypes. The coordinates inferred by such approaches tend to be highly correlated with the geographic locations from where individuals were sampled (Novembre et al., 2008; Wang et al., 2012). EIGENSTRAT (Patterson et al., 2006; Price et al., 2006) is a well-known program that implements PCA.

One of the issues with methods such as STRUCTURE and ADMIXTURE is that they only consider individual markers and not their joint variation patterns. Markers on the same chromosome tend to be inherited together in the absence of recombination. For close markers, at a population level, this results in LD, i.e., non-random associations that reflects shared genealogy and invalidates the independence assumption. For dense polymorphism datasets such as those obtained from sequencing, haplotype based analysis has the potential to leverage this information and provide improved ability to detect population substructure. ChromoPainter and fineSTRUCTURE (Lawson et al., 2012) are recently developed programs that aim to make use of haplotype structure for high quality PCA and population structure inference respectively. The modeling of $\mathrm{LD}$ leads to more accurate structure inference but at a cost of significantly higher running times as compared to programs such as PCA and ADMIXTURE.

\section{METHODS FOR LOCAL ANCESTRY INFERENCE}

Additional complexities to ancestry inference can occur when a population arises as a product of two or more divergent populations mixing for a certain period of time (i.e., admixed populations such as African Americans and Latinos). GA inference methods will assign every individual in such populations to more than one group. Admixed genomes are mosaics of segments originating from different ancestral populations and estimating the ancestral proportions and in particular, finding the regional ancestry at each genomic location in such situations is a particularly challenging problem. Most of the methods that have been developed so far take a generative approach to solve this and try to fit an explicit probabilistic model to the data using a hidden Markov model (HMM) or its extensions. Generative approaches for LA inference first try to model the joint dependence of alleles and ancestry and subsequently use "Bayes" rule to estimate the dependence of ancestry on SNP allele configurations.

Early approaches to LA inference based on the STRUCTURE framework (Falush et al., 2003; Hoggart et al., 2004; Patterson et al., 2004) made use of HMMs and did not explicitly model background LD. One limitation of such methods is that they do not fully leverage the information that is available in haplotypes which can potentially be useful for distinguishing closely related populations. In contrast other methods that can explicitly model LD [e.g., SABER: Tang et al., 2006; HAPAA (HMM-based analysis of polymorphisms in admixed ancestries): Sundquist et al., 2008; HAPMIX: Price etal., 2009] are computationally intensive and are able to consider only two ancestral populations at a time. LAMP (local ancestry in admixed populations) is a state of the art algorithm for estimation of LA in recently admixed populations (Sankararaman etal., 2008) that operates on sliding windows of contiguous SNPs and assigns ancestries based on a clustering algorithm. It was shown to be more accurate and significantly faster than STRUCTURE $\left(\sim 10^{4}\right.$ times faster $)$ and SABER ( $\sim 200$ times faster). One of the underlying assumptions is the absence of recombination within windows. WINPOP is a modification of the original LAMP framework that uses a refined model of recombination events and an efficient dynamic programming algorithm to improve LA inference for situations where ancestral populations are closely related (Pasaniuc et al., 2009). PCAdmix (Brisbin, 2010) is a heuristic approach for LA inference. This approach first divides the genome into windows 
of $10-50 \mathrm{~kb}$ width and estimates the probability of origin from particular reference panel populations using PCA. These probabilities are then used as emission probabilities in a HMM to infer ancestry via Viterbi decoding. SupportMix is another recently developed approach for LA inference that trains Support Vector Machines in a sliding window HMM framework (Omberg et al., 2012). ASPCA (ancestry-specific principal components analysis ) is a novel method for inferring the within-continental origin of haplotypes along the genome for admixed populations and was developed recently in the context of reconstructing the history of Caribbean populations (Moreno-Estrada et al., 2013). It involves first inferring LA and then applying PCA to only genomic segments of specific ancestries.

An alternative approach to LA inference, RFMix was developed recently which takes a discriminative approach to this problem (Maples et al., 2013). Such approaches model the dependency of the unobserved variables (i.e., ancestries) directly as a function of the observed variables (i.e., alleles). RFMix makes use of conditional random fields which are based on random forests trained on reference panels. LA inference based on RFMix was shown to be faster and more accurate than many competing approaches such as LAMP ( $\sim 33$ fold faster) and Support Mix $(\sim 1.7$ fold faster). EILA (efficient inference of local ancestry; Yang et al., 2013) is another recently developed statistical method that uses fused quantile regression and a $k$-means classifier to perform LA inference. The method does not assume linkage equilibrium between markers and proposes to use all the genotyped SNPs for greater accuracy. EILA has been shown to be more accurate than programs such as LAMP and HAPMIX when the ancestral distance between populations is large or moderate and is comparable in terms of speed.

\section{NUMBER OF SOURCE POPULATIONS AND ANCESTRAL MISSPECIFICATION}

Many LA inference methods assume that the ancestral source populations as well as their allele frequencies are known and use these as inputs. In practice, such information may either not be available or even if available could be inaccurate (e.g., for Native Americans). Simulation experiments demonstrate that errors in ancestral specification can significantly impact LA prediction accuracy and the drop in accuracy is higher for closely related ancestral populations (Pasaniuc et al., 2009). Thus, choosing accurate ancestral groups is crucial for such scenarios but is less critical when ancestral groups are distant (Pasaniuc et al., 2009). In the absence of ancestral population information, many existing approaches can also utilize the information contained in the admixed samples themselves to estimate LA de novo (e.g., Sankararaman et al., 2008; Maples et al., 2013).

Most of these previously mentioned methods have been demonstrated to be highly accurate for the case of two way admixtures such as in African Americans (Seldin et al., 2011). However, the accuracy of such methods declines for more complicated scenarios such as the admixture of three ancestral populations in case of Latinos (European, African, and Native American). The presence of closely related populations in multi-way admixtures (e.g., Europeans and Native Americans) further increases the difficulty of inference. Many existing methods either cannot handle these scenarios or are prone to high error rates making it hard to reliably study LA in such cases. Keeping these issues in mind, several new approaches were developed in the last few years to more effectively handle multi-way admixtures. Johnson et al. (2011) use an extension of SABER to three-way mixtures in a haploid mode to infer virtual genomes. Henn et al. (2012) extended the work of Bryc etal. (2010) to employ PCA and HMMs to estimate ancestries for multi-way admixtures. LAMPLD and LAMP-HAP (Baran et al., 2012) are extensions of the LAMP algorithm designed for dealing with multi-way admixtures and combine HMMs with an innovative window-based framework to achieve high accuracy estimates in Latinos. Rodriguez et al. (2013) describe a LA inference method ALLOY that utilizes a factorial HMM to capture the process generating maternal and paternal admixed haplotypes, and, inhomogeneous variable length Markov Chains to model the background LD in ancestral populations. ALLOY can handle both recent and ancient admixtures with up to four ancestral populations. Guan (2014) presented a two-layer HMM to detect structure of local haplotypes and demonstrated its utility for LA inference for both two-way and three-way admixture. Lanc-CSV (local ancestry using continent specific variants) is a new method for ultra-fast and accurate inference of LA in very large sequenced cohorts by using continent specific variants in a standard HMM framework (Brown and Pasaniuc, 2014).

\section{APPLICATIONS OF GLOBAL AND LOCAL ANCESTRY INFERENCE}

Ancestry estimation using genomic data has proven to be very useful for many different applications. Importantly, in genetic association studies, ancestry inference can be used to account for the effects of population stratification which is a serious confounding factor and can lead to elevated rates of false positives (Price et al., 2010). In many scenarios, one is interested in the presence of "cryptic" population structure, i.e., structure that is significant and detectable only in genetic terms and not by external features. Estimation of cryptic population structure is also important for DNA fingerprinting to quantify the probability of false matches (Balding and Nichols, 1994, 1995; Foreman et al., 1997; Roeder et al., 1998).

Global ancestry inference is also useful in many evolutionary studies, where we are interested in learning more about the properties of populations and the relationships among them (Cavalli-Sforza et al., 1994). For this purpose, it is useful if we can classify samples into populations. Similarly, given the knowledge of different population groups, one may wish to classify an individual of unknown origin into one of these groups (Davies et al., 1999) or determine if an individual is an immigrant. In the personal genomics space, many private companies now provide ancestry testing products which make use of genome-wide markers from individuals (Royal et al., 2010). This can enable individuals to learn more about the details of their ancestral history and geographical origins. Lastly, GA inference methods have also proven useful for inferring population structure in many non-human species such as maize (Zea mays; Pritchard, 2001), chickens (Gallus gallus domesticus; Rosenberg et al., 2001), thale cress (Arabidopsis thaliana; Nordborg et al., 2005), rice (Oryza 
sativa; McNally et al., 2009), and chimpanzees (Pan troglodytes; Becquet et al., 2007).

Like GA inference, LA inference has also found numerous applications. The most important application of LA inference has been to map genes to disease through admixture mapping in populations such as African Americans and Latinos (e.g., Hoggart etal., 2004; Zhu et al., 2004; Reich et al., 2005; Seldin et al., 2011). Other crucial applications have included pharmacogenomics; for example, in a recent study, Native American ancestry was significantly associated with the risk of relapse in children suffering from acute lymphoblastic leukemia (Yang et al., 2011). In addition to these traditional applications, in the more recent years, LA inference methods have also found applications in other settings such as localizing sequences of unknown location from the human reference genome (Genovese et al., 2013), studying recombination rate variation (Hinch et al., 2011; Wegmann et al., 2011), inferring natural selection (Tang et al., 2007; Jin et al., 2012), making demographic inferences (Bryc et al., 2010; Johnson et al., 2011; Kidd et al., 2012), and in joint association and admixture mapping to boost the power to detect disease linked genes and variants (Pasaniuc et al., 2011; Shriner et al., 2011).

\section{FUTURE RESEARCH AND CHALLENGES IN ANCESTRY INFERENCE}

With rapid advances in sequencing technologies, the amount of genomic data available to us has grown massively in the recent years. With the advent of dense variation data from fully sequenced samples of genomes in thousands of individuals (e.g., 1000 genomes project) and advances in haplotype phasing methods, we can anticipate new ancestry inference methods as well as refinement of existing ones to more fully exploit the information available. How to maximally utilize the rich information available in the form of haplotypes in such exhaustive catalogs of variation while developing inference methods that are also computationally efficient and scalable for large sample sizes is an important challenge for the future. For both global and LA methods, there is also scope for improved modeling of background LD between genetic variants that can lead to lower error rates and enhance our ability to detect subtle kinds of population structure. The availability of large genomic datasets also allows us to characterize the geographic locations of individuals with unprecedented detail and more effectively distinguish between closely related population groups. More accurate tools for population structure inference will therefore also lead to more reliable knowledge of the ancestral history for individuals in personal genomics and better facilitate personalized medicine. Similarly, improved methods for LA inference based on such large datasets are also likely to generate more powerful tools for admixture mapping particularly for populations with complex admixture history.

\section{REFERENCES}

Alexander, D. H., Novembre, J., and Lange, K. (2009). Fast model-based estimation of ancestry in unrelated individuals. Genome Res. 19, 1655-1664. doi: 10.1101/gr.094052.109

Balding, D. J., and Nichols, R. A. (1994). DNA profile match probability calculations: how to allow for population stratification, relatedness, database selection and single bands. Forensic Sci. Int. 64, 125-140. doi: 10.1016/0379-0738(94) 90222-4

Balding, D. J., and Nichols, R. A. (1995). A method for quantifying differentiation between populations at multi-allelic loci and its implications for investigating identity and paternity. Genetica 96, 3-12. doi: 10.1007/BF014 41146

Baran, Y., Pasaniuc, B., Sankararaman, S., Torgerson, D. G., Gignoux, C., Eng, C., et al. (2012). Fast and accurate inference of local ancestry in Latino populations. Bioinformatics 28, 1359-1367. doi: 10.1093/bioinformatics/bts144

Becquet, C., Patterson, N., Stone, A. C., Przeworski, M., and Reich, D. (2007). Genetic structure of chimpanzee populations. PLoS Genet. 3:e66. doi: 10.1371/journal.pgen.0030066

Bouaziz, M., Paccard, C., Guedj, M., and Ambroise, C. (2012). SHIPS: spectral hierarchical clustering for the inference of population structure in genetic studies. PLoS ONE 7:e45685. doi: 10.1371/journal.pone.0045685

Brisbin, A. (2010). Linkage Analysis for Categorical Traits and Ancestry Assignment in Admixed Individuals. Ph.D. thesis, Cornell University, Ithaca.

Brown, R., and Pasaniuc, B. (2014). Enhanced methods for local ancestry assignment in sequenced admixed individuals. PLoS Comput. Biol. 10:e1003555. doi: 10.1371/journal.pcbi.1003555

Bryc, K., Auton, A., Nelson, M. R., Oksenberg, J. R., Hauser, S. L., Williams, S., et al. (2010). Genome-wide patterns of population structure and admixture in West Africans and African Americans. Proc. Natl. Acad. Sci. U.S.A. 107, 786-791. doi: 10.1073/pnas.0909559107

Cavalli-Sforza, L. L., Menozzi, P., and Piazza, A. (1994). The History and Geography of Human Genes. Princeton, NJ: Princeton University Press.

Davies, N., Villablanca, F. X., and Roderick, G. K. (1999). Determining the source of individuals: multilocus genotyping in nonequilibrium population genetics. Trends Ecol. Evol. 14, 17-21. doi: 10.1016/S0169-5347(98)01530-4

Falush, D., Stephens, M., and Pritchard, J. K. (2003). Inference of population structure using multilocus genotype data: linked loci and correlated allele frequencies. Genetics 164, 1567-1587.

Foreman, L., Smith, A., and Evett, I. (1997). Bayesian analysis of DNA in forensic identification applications. J. R. Stat. Soc. A 160, 429-469. doi: 10.1111/j.1467985X.1997.00074.x

Frichot, E., Mathieu, F., Trouillon, T., Bouchard, G., and François, O. (2014). Fast and efficient estimation of individual ancestry coefficients. Genetics 196, 973-983. doi: 10.1534/genetics.113.160572

Gao, H., Williamson, S., and Bustamante, C. D. (2007). A Markov chain Monte Carlo approach for joint inference of population structure and inbreeding rates from multilocus genotype data. Genetics 176, 1635-1651. doi: 10.1534/genetics.107.072371

Gao, X., and Starmer, J. (2007). Human population structure detection via multilocus genotype clustering. BMC Genet. 8:34. doi: 10.1186/1471-2156-8-34

Genovese, G., Handsaker, R. E., Li, H., Altemose, N., Lindgren, A. M., Chambert, K., et al. (2013). Using population admixture to help complete maps of the human genome. Nat. Genet. 45, 406-414. doi: 10.1038/ng.2565

Guan, Y. (2014). Detecting structure of haplotypes and local ancestry. Genetics 196, 625-642. doi: 10.1534/genetics.113.160697

Henn, B. M., Botigué, L. R., Gravel, S., Wang, W., Brisbin, A., Byrnes, J. K., et al. (2012). Genomic ancestry of North Africans supports back-to-Africa migrations. PLoS Genet. 8:e1002397. doi: 10.1371/journal.pgen.1002397

Hinch, A., Tandon, A., Patterson, N., Song, Y., Rohland, N., Palmer, C. D., et al. (2011). The landscape of recombination in African Americans. Nature 476, 170175. doi: $10.1038 /$ nature 10336

Hoggart, C. J., Shriver, M. D., Kittles, R. A., Clayton, D. G., and McKeigue, P. M. (2004). Design and analysis of admixture mapping studies. Am. J. Hum. Genet. 74, 965-978. doi: 10.1086/420855

Jin, W., Xu, S., Wang, H., Yu, Y., Shen, Y., Wu, B., et al. (2012). Genome-wide detection of natural selection in African Americans pre- and post-admixture. Genome Res. 22, 519-527. doi: 10.1101/gr.124784.111

Johnson, N. A., Coram, M. A., Shriver, M. D., Romieu, I., Barsh, G. S., London, S. J., et al. (2011). Ancestral components of admixed genomes in a Mexican cohort. PLoS Genet. 7:e1002410. doi: 10.1371/journal.pgen.1002410

Kidd, J. M., Gravel, S., Byrnes, J., Moreno-Estrada, A., Musharoff, S., Bryc, K., et al. (2012). Population genetic inference from personal genome data: impact of ancestry and admixture on human genomic variation. Am. J. Hum. Genet. 91, 660-671. doi: 10.1016/j.ajhg.2012.08.025 
Lao, O., Liu, F., Wollstein, A., and Kayser, M. (2014). GAGA: a new algorithm for genomic inference of geographic ancestry reveals fine level population substructure in Europeans. PLoS Comput. Biol. 10:e1003480. doi: 10.1371/journal.pcbi.1003480

Lawson, D. J., Hellenthal, G., Myers, S., and Falush, D. (2012). Inference of population structure using dense haplotype data. PLoS Genet. 8:e1002453. doi: 10.1371/journal.pgen.1002453

Lee, C., Abdool, A., and Huang, C. H. (2009). Pca-based population structure inference with generic clustering algorithms. BMC Bioinformatics 10(Suppl. 1):S73. doi: 10.1186/1471-2105-10-S1-S73

Maples, B. K., Gravel, S., Kenny, E. E., and Bustamante, C. D. (2013). RFMix: a discriminative modeling approach for rapid and robust local-ancestry inference. Am. J. Hum. Genet. 93, 278-288. doi: 10.1016/j.ajhg.2013.06.020

McNally, K. L., Childs, K. L., Bohnert, R., Davidson, R. M., Zhao, K., Ulat, V. J., et al. (2009). Genomewide SNP variation reveals relationships among landraces and modern varieties of rice. Proc. Natl. Acad. Sci. U.S.A. 106, 12273-12278. doi: 10.1073/pnas.0900992106

Moreno-Estrada, A., Gravel, S., Zakharia, F., McCauley, J. L., Byrnes, J. K., Christopher, R., etal. (2013). Reconstructing the population genetic history of the Caribbean. PLoS Genet. 9:e1003925. doi: 10.1371/journal.pgen.1003925

Nordborg, M., Hu, T. T., Ishino, Y., Jhaveri, J., Toomajian, C., Zheng, H., et al. (2005). The pattern of polymorphism in Arabidopsis thaliana. PLoS Biol. 3:e196. doi: 10.1371/journal.pbio.0030196

Novembre, J., Johnson, T., Bryc, K., Kutalik, Z., Boyko, A. R., Auton, A., et al. (2008). Genes mirror geography within Europe. Nature 456, 98-101. doi: 10.1038 /nature07331

Omberg, L., Salit, J., Hackett, N., Fuller, J., Matthew, R., Chouchane, L., et al. (2012) Inferring genome-wide patterns of admixture in Qataris using fifty-five ancestral populations. BMC Genet. 13:49. doi: 10.1186/1471-2156-13-49

Pasaniuc, B., Sankararaman, S., Kimmel, G., and Halperin, E. (2009). Inference of locus-specific ancestry in closely related populations. Bioinformatics 25, i213i221. doi: 10.1093/bioinformatics/btp197

Pasaniuc, B., Zaitlen, N., Lettre, G., Chen, G. K., Tandon, A., Kao, W. H., et al. (2011). Enhanced statistical tests for GWAS in admixed populations: assessment using African Americans from CARe and a Breast Cancer Consortium. PLoS Genet 7:e1001371. doi: 10.1371/journal.pgen.1001371

Patterson, N., Hattangadi, N., Lane, B., Lohmueller, K. E., Hafler, D. A., Oksenberg, J. R., et al. (2004). Methods for high-density admixture mapping of disease genes. Am. J. Hum. Genet. 74, 979-1000. doi: 10.1086/420871

Patterson, N., Price, A., and Reich, D. (2006). Population structure and eigenanalysis. PLoS Genet. 2:e190. doi: 10.1371/journal.pgen.0020190

Price, A. L., Tandon, A., Patterson, N., Barnes, K. C., Rafaels, N., Ruczinski, I., et al. (2009). Sensitive detection of chromosomal segments of distinct ancestry in admixed populations. PLoS Genet. 5:e1000519. doi: 10.1371/journal.pgen.1000519

Price, A. L., Zaitlen, N. A., Reich, D., and Patterson, N. (2010). New approaches to population stratification in genome-wide association studies. Nat. Rev. Genet. 11, 459-463. doi: 10.1038/nrg2813

Price, A. L., Patterson, N., Plenge, R., Weinblatt, M., Shadick, N., and Reich, D. (2006). Principal components analysis corrects for stratification in genome-wide association studies. Nat. Genet. 38, 904-909. doi: 10.1038/ng1847

Pritchard, J. K. (2001). Deconstructing maize population structure. Nat. Genet. 28 , 203-204. doi: 10.1038/90026

Pritchard, J. K., Stephens, M., and Donnelly, P. (2000). Inference of population structure using multilocus genotype data. Genetics 155, 945-959.

Purcell, S., Neale, B., Todd-Brown, K., Thomas, L., Ferreira, M. A., Bender, D., et al. (2007). PLINK: a tool set for whole-genome association and population- based linkage analyses. Am. J. Hum. Genet. 81, 559-575. doi: 10.1086/519795

Raj, A., Stephens, M., and Pritchard, J. K. (2014). fastSTRUCTURE: variational inference of population structure in large SNP datasets. Genetics 114:164350. doi: 10.1534/genetics.114.164350

Reich, D., Patterson, N., De Jager, P. L., McDonald, G. J., Waliszewska, A., Tandon, A., et al. (2005). A whole-genome admixture scan finds a candidate locus for multiple sclerosis susceptibility. Nat. Genet. 37, 1113-1118. doi: 10.1038/ng1646

Rodriguez, J. M., Bercovici, S., Elmore, M., and Batzoglou, S. (2013). Ancestry inference in complex admixtures via variable-length Markov chain linkage models. J. Comput. Biol. 20, 199-211. doi: 10.1089/cmb.2012.0088
Roeder, K., Escobar, M., Kadane, J. B., and Balazs, I. (1998). Measuring heterogeneity in forensic databases using hierarchical Bayes models. Biometrika 85, 269-287. doi: 10.1093/biomet/85.2.269

Rosenberg, N. A., Burke, T., Elo, K., Feldman, M. W., Friedlin, P., Groenen, M. A., et al. (2001). Empirical evaluation of genetic clustering methods using multilocus genotypes from 20 chicken breeds. Genetics 159, 699-713.

Rosenberg, N. A., Pritchard, J. K., Weber, J. L., Cann, H. M., Kidd, K. K., Zhivotovsky, L. A., et al. (2002). Genetic structure of human populations. Science 298, 23812385. doi: 10.1126/science. 1078311

Royal, C. D., Novembre, J., Fullerton, S. M., Goldstein, D. B., Long, J. C., Bamshad, M. J., et al. (2010). Inferring genetic ancestry: opportunities, challenges, and implications. Am. J. Hum. Genet. 86, 661-673. doi: 10.1016/j.ajhg.2010. 03.011

Sankararaman, S., Sridhar, S., Kimmel, G., and Halperin, E. (2008). Estimating local ancestry in admixed populations. Am. J. Hum. Genet. 82, 290-303. doi: 10.1016/j.ajhg.2007.09.022

Seldin, M., Pasaniuc, B., and Price, A. L. (2011). New approaches to disease mapping in admixed populations. Nat. Rev. Genet. 12, 523-528. doi: 10.1038/nrg3002

Shriner, D., Adeyemo, A., and Rotimi, C. N. (2011). Joint ancestry and association testing in admixed individuals. PLoS Comput. Biol. 7:e1002325. doi: 10.1371/journal.pcbi.1002325

Sundquist, A., Fratkin, E., Do, C. B., and Batzoglou, S. (2008). Effect of genetic divergence in identifying ancestral origin using HAPAA. Genome Res. 18, 676-682. doi: 10.1101/gr.072850.107

Tang, H., Choudhry, S., Mei, R., Morgan, M., Rodriguez-Cintron, W., Burchard, E., et al. (2007). Recent genetic selection in the ancestral admixture of Puerto Ricans. Am. J. Hum. Genet. 81, 626-633. doi: 10.1086/520769

Tang, H., Coram, M., Wang, P., Zhu, X., and Risch, N. (2006). Reconstructing genetic ancestry blocks in admixed individuals. Am. J. Hum. Genet. 79, 1-12. doi: $10.1086 / 504302$

Tang, H., Peng, J., Wang, P., and Risch, N. (2005). Estimation of individual admixture: analytical and study design considerations. Genet. Epidemiol. 28, 289-301. doi: 10.1002/gepi.20064

Wang, C., Zöllner, S., and Rosenberg, N. A. (2012). A quantitative comparison of the similarity between genes and geography in worldwide human populations. PLoS Genet. 8:e1002886. doi: 10.1371/journal.pgen.1002886

Wegmann, D., Kessner, D., Veeramah, K., Mathias, R., Nicolae, D., Yanek, L., et al. (2011). Recombination rates in admixed individuals identified by ancestry-based inference. Nat. Genet. 43, 847-853. doi: 10.1038/ng.894

Yang, J., Cheng, C., Devidas, M., Cao, X., Fan, Y., Campana, D., et al. (2011). Ancestry and pharmacogenomics of relapse in acute lymphoblastic leukemia. Nat. Genet. 43, 237-241. doi: 10.1038/ng.763

Yang, J. J., Li, J., Buu, A., and Williams, L. K. (2013). Efficient inference of local ancestry. Bioinformatics 29, 2750-2756. doi: 10.1093/bioinformatics/ btt488

Yang, W.-Y., Novembre, J., Eskin, E., and Halperin, E. (2012). A model based approach for analysis of spatial structure in genetic data. Nat. Genet. 44, 725-731. doi: $10.1038 / \mathrm{ng} .2285$

Zhu, X., Cooper, R. S., and Elston, R. C. (2004). Linkage analysis of a complex disease through use of admixed populations. Am. J. Hum. Genet. 74, 1136-1153. doi: $10.1086 / 421329$

Conflict of Interest Statement: The author declares that the research was conducted in the absence of any commercial or financial relationships that could be construed as a potential conflict of interest.

Received: 29 April 2014; accepted: 17 June 2014; published online: 03 July 2014 Citation: Padhukasahasram B (2014) Inferring ancestry from population genomic data and its applications. Front. Genet. 5:204. doi: 10.3389/fgene.2014.00204

This article was submitted to Evolutionary and Population Genetics, a section of the journal Frontiers in Genetics.

Copyright (c) 2014 Padhukasahasram. This is an open-access article distributed under the terms of the Creative Commons Attribution License (CC BY). The use, distribution or reproduction in other forums is permitted, provided the original author(s) or licensor are credited and that the original publication in this journal is cited, in accordance with accepted academic practice. No use, distribution or reproduction is permitted which does not comply with these terms. 